\title{
Informations
}

\section{"FLOTROP", une base de données agropastorales sur l'Afrique tropicale au CIRAD-EMVT}

En 1958, le Docteur Pagot a créé à l'IEMVT une section chargée de l'étude de la végétation en tant que ressource alimentaire du bétail africain. Depuis, les agropastoralistes de terrain ont arpenté l'Afrique tropicale en tous sens et, sous l'impulsion de G. Boudet, ont enregistré des observations floristiques par dizaines de milliers dans un nombre très élevé de relevés largement répartis du Cap-Vert à Djibouti et de la zone guinéenne à la zone saharienne. Plus de 3,5 millions d'hectares sont ainsi concernés par une grande série de rapports décrivant les formations végétales, leurs usages, leur valeur pastorale, leurs possibilités d'amélioration. Une synthèse des connaissances a été faite dans une série d'atlas à moyenne échelle ${ }^{1,2}:$ «Elevage et potentialités pastorales sahéliennes».

Par ailleurs, les collections botaniques effectuées à l'occasion de ces tournées ont permis la constitution d'un herbier de référence qui fait autorité pour les régions sahéliennes. Cet herbier va prochainement être installé dans les locaux neufs du nouveau campus du CIRAD à Baillarguet, près de Montpellier. En complément de la mise sur une base de données de la nomenclature et des collectes, déja faite ${ }^{3}$, les aménagements et équipements réunis à cette occasion feront de cette collection de référence une des mieux installées et des plus modernes de France. La codification des espèces sahéliennes initiée par $G$. Boudet sert d'ores et déjà de référence auprès des utilisateurs, comme la mise au point nomenclaturale par Lebrun et Stork ${ }^{4}$,en cours d'achèvement, constitue désormais une référence internationale incontournable.

Si l'on ajoute aux relevés propres des agrostologues du CIRAD-EMVT ceux des chercheurs d'autres instituts (Muséum national d'histoire naturelle, Universités), les observations faites en Afrique tropicale paléarctique sont en nombre considérable et s'étalent sur les 60 dernières années.

Le projet FLOTROP ${ }^{5}$, mis en place par le CIRAD-EMVT avec l'aide du CNRS, a pour objectif de rassembler cette masse de données, d'en homogénéiser la présentation et la nomenclature et d'intégrer le corpus ainsi réuni dans une base de données floristiques et agrostologiques. II porte sur un territoire qui s'étend sur $80^{\circ}$ de longitude, de $25^{\circ}$ ouest à $55^{\circ}$ est et sur $20^{\circ}$ de latitude de $5^{\circ}$ nord à $25^{\circ}$

1. Elevage et potentialités pastorales sahéliennes. Wageningen, Pays-Bas, CTA ; Maisons-Alfort, France, CIRAD-IEMVT.

2. GASTON A. (ed.), 1994. Les pâturages sahéliens de l'Afrique de l'Ouest. Wageningen, Pays-Bas, CTA ; Maisons-Alfort, France, CIRAD-EMVT, $220 \mathrm{p}$

3. ROY F., 1993. Structure de la base de données botaniques du CIRAD-EMVT. Maisons-Alfort, France, CIRAD-EMVT, $18 \mathrm{p}$.

4. LEBRUN J.P., STORK A.,1991, 1992, 1995. Enumération des plantes à fleurs d'Afrique tropicale. Genève, Suisse, Conservatoire et Jardin botanique, 250 p., 258 p., $300 p$.

5. TACHER G., 1993. Le projet FLOTROP au CIRAD-EMVT Flotrop info, $1: 1-2$ nord, donc du Hoggar au golfe de Guinée et des lles du Cap-Vert à la corne de l'Afrique. Cette portion de l'Afrique comporte 1600 degrés-carré. Elle a été divisée en 18 territoires qui peuvent correspondre à un seul pays ou en réunir plusieurs contigus lorsque leurs dimensions sont plus réduites:

$\begin{array}{ll}1 \text { Algérie } & 10 \text { Mauritanie } \\ 2 \text { Bénin } & 11 \text { Mali } \\ 3 \text { Burkina Faso } & 12 \text { Niger } \\ 4 \text { Cameroun } & 13 \text { Sénégal, Gambie } \\ 5 \text { Cap-Vert } & 14 \text { Soudan } \\ 6 \text { Centrafrique } & 15 \text { Tchad } \\ 7 \text { Côte d'Ivoire } & 16 \text { Ethiopie, Somalie } \\ 8 \text { Djibouti, Erythrée } & 17 \text { Nigeria } \\ 9 \text { Guinées, Liberia } & 18 \text { Togo, Ghana }\end{array}$

Si la base doit rassembler le plus possible de relevés, elle ne peut incorporer n'importe quelle observation ! II y a des conditions à remplir pour qu'un relevé puisse être accepté et intégré.

- Tout d'abord, un relevé doit être daté avec autant de précision que possible. Chacun connait les différences considérables existant entre les phénophases des formations sahéliennes, c'est-à-dire entre les aspects que peut présenter la physionomie d'une végétation sahélienne en fin de saison sèche et au cœur de la saison des pluies. Pour que la banque de données permette d'aborder des études comparatives précises, et surtout dans le cas de suivi, il faut être en mesure de localiser les observations utilisées par rapport aux dates du début des saisons sèche et pluvieuse, des premières "pluies efficaces", en fonction de la durée de la saison des pluies, du total des précipitations déjà reçues et du module pluviométrique, alors que tous ces paramètres changent d'une manière importante d'une année à l'autre.

- Ensuite, un relevé doit être géoréférencé, c'est-à-dire localisé dans l'espace avec une précision suffisante pour permettre le rapprochement de deux observations faites en des emplacements très voisins par des observateurs différents et à des dates pouvant être éloignées ; c'est aussi la contrainte qui garantit la validité des cartes de répartition permises par la banque de données. L'idéal est que l'observateur localise ses observations avec un GPS, mais pour les relevés anciens, c'est impossible. Certains observateurs, ou certaines études particulièrement soignées, donnent les coordonnées précises des emplacements de leurs relevés; d'autres permettent d'en 
retrouver les valeurs par des cartes de localisation. Certaines, seulement verbales, sont alors très difficiles à positionner sur une carte et laissent un "déchet" de 25 à 50 p. 100.

- Enfin, un relevé doit être signé. D'abord, pour que les travaux de synthèse qui peuvent ètre faits à partir de la base FLOTROP puissent rendre à chacun son dû. Ensuite, parce que la connaissance de l'identité du collecteur peut faciliter les procédures de transcription nomenclaturale, expliquer des anomalies apparentes de biodiversité (les aires analysées, dites aires minimales, sont très différentes d'un collecteur à l'autre dans le même type de végétation), permettre de remonter aux sources lorsque des cas de singularités apparaissent.

Un outil informatique d'analyse et de gestion de ces données est développé dans le cadre du laboratoire de Botanique de l'EMVT à Montpellier. Il a pour fonction de permettre la validité des données à entrer, d'en assurer l'incorporation et la maintenance et d'en permettre l'utilisation.

Cet outil permet la reprise aisée de tableaux phytosociologiques et leur réorganisation en relevés individualisés; il permet la codification rapide de liste d'espèces et la "décodification" de séries de codes. Les codes utilisés antérieurement à l'EMVT sont conservés, mais la liste en a été complétée par la codification des genres et par l'addition progressive de codes nouveaux pour les espèces nouvellement rencontrées dans les listes incorporées, toujours en référence à la nomenclature de Lebrun et Stork ${ }^{4}$ et des synonymes aux binoms de ces auteurs.

Les entrées sont assorties d'un système de contrôle qui vérifie la cohérence des valeurs tapées avant de les accepter et d'un système de correction permettant de modifier chacun des éléments enregistrés. Ces enregistrements sont traités et répartis dans une série de fichiers dont la spécialisation permet une exploitation rapide ; de plus, chaque enregistrement est directement accessible quelle que soit sa place dans les fichiers par sa structure même, ce qui permet encore d'accélérer les accès aux données.
Contrairement à beaucoup de systèmes développés dans d'autres institutions, FLOTROP n'est pas destiné à effectuer directement des traitements statistiques ou des "analyses de données". FLOTROP comporte des modules de sortie générant des fichiers «ASCll» dont les formats permettent la reprise par les progiciels.

Par contre, les divers types de sortie font l'objet d'une attention particulière. Outre ceux qui viennent d'être évoqués, il est possible d'obtenir une édition standardisée des relevés dans la nomenclature de référence (celle de la note 4). ; d'obtenir la florule d'un degré-carré, d'un groupe de degrés-carré contigus (une fenêtre) ou d'un territoire entier et d'opter pour un classement des espèces par ordre de code (par défaut), par ordre alphabétique, ou par famille; d'extraire la liste des relevés d'un degré-carré, d'une fenêtre, d'un auteur dans un pays, contenant une espèce particulière dans un territoire; de cartographier à 3' près l'ensemble des relevés de la dition, ou seulement ceux qui contiennent une espèce particulière, ou l'ensemble des relevés d'un degré-carré (localisés à 1 ' près sur une carte au millionième), ou encore l'ensemble des relevés d'un territoire contenant une espèce particulière. Toutes ces sorties cartographiques sont accompagnées de sorties sur des fichiers «ASCIl» des données exploitées pour permettre leur incorporation dans des rapports.

Le progiciel FLOTROP est prévu pour intégrer les références des cartes écologiques ou agrostologiques pertinentes et afficher le liste des documents concernés lors d'interrogations par degré-carré. II permettra, dans une étape ultérieure, des interprétations agrostologiques des relevés incorporés : construction du diagramme pastoral, des spectres biologiques, de la biodiversité, mise en évidence des potentialités pastorales. II permettra aussi l'édition de fiches spécifiques sur les caractéristiques des espèces pastorales.

Un bulletin bimestriel, Flotrop info, est diffusé par le laboratoire de Botanique de l'EMVT pour traduire et suivre l'état d'avancement des travaux et de l'enrichissement de la base.

\author{
Philippe DAGET \\ CNRS - CIRAD-EMVT
}

